\title{
Generalized anxiety disorder in type 2 diabetes mellitus: prevalence and clinical characteristics
}

\author{
Chun-Jen Huang, ${ }^{1,2}$ Hui-Min Hsieh, ${ }^{3}$ Hung-Pin Tu, ${ }^{4}$ He-Jiun Jiang, ${ }^{5}$ Peng-Wei Wang, ${ }^{1,2}$ \\ Ching-Hua Lin ${ }^{2,6}$ (iD \\ ${ }^{1}$ Department of Psychiatry, Kaohsiung Medical University Hospital, Kaohsiung, Taiwan. ${ }^{2}$ Department of Psychiatry, Faculty of Medicine, \\ College of Medicine, Kaohsiung Medical University, Kaohsiung, Taiwan. ${ }^{3}$ Department of Public Health, Kaohsiung Medical University, \\ Kaohsiung, Taiwan. ${ }^{4}$ Department of Public Health and Environmental Medicine, School of Medicine, College of Medicine, Kaohsiung Medical \\ University, Kaohsiung, Taiwan. ${ }^{5}$ Division of Endocrinology and Metabolism, Department of Internal Medicine, Kaohsiung Medical University \\ Hospital, Kaohsiung Medical University, Kaohsiung, Taiwan. ${ }^{6}$ Department of Adult Psychiatry, Kai-Syuan Psychiatric Hospital, Kaohsiung, \\ Taiwan.
}

\begin{abstract}
Objective: This study investigated the prevalence of generalized anxiety disorder (GAD) in Taiwanese patients with type 2 diabetes mellitus (T2DM).

Methods: This retrospective observational study was conducted with a random sample of patients from the entire population of National Health Insurance enrollees during 2000-2010 and used ICD-9CM diagnostic codes to identify T2DM patients and GAD. The prevalence of GAD was compared between T2DM patients and the general population.

Results: Between 2000 and 2010, the prevalence of GAD was significantly greater in the T2DM patients than the general population, while the increase of GAD was higher in the general population (from 0.25 to $0.63 \%$ ) than among T2DM patients (from 0.81 to $1.03 \%$ ). In T2DM patients, GAD was associated with female gender, a Charlson Comorbidity Index $\geqslant 1$, diabetes mellitus duration $>9$ years, and the following comorbidities: congestive heart failure, peripheral vascular disease, and depressive disorder. The prevalence of GAD among T2DM patients was negatively associated with rapid-acting insulin injection therapy and with the use of metformin and sulfonylureas.

Conclusion: Since the prevalence of GAD was greater among T2DM patients than the general population, public health initiatives are needed to prevent and treat GAD in T2DM patients, specifically those with the above mentioned risk factors.
\end{abstract}

Keywords: National health insurance; prevalence; generalized anxiety disorder; type 2 diabetes mellitus

\section{Introduction}

Generalized anxiety disorder (GAD) is among the most prevalent mental health conditions and is characterized by excessive and uncontrollable worry that leads to impairment or significant distress. ${ }^{1}$ The estimated 12-month prevalence of GAD is $3.9 \%$ (range, 2.1-6.6\%), and the estimated lifetime prevalence of GAD is $12 \%$ (range, $8-13.7 \%)^{2}$ Notably, $45-91 \%$ of GAD patients have comorbidities, such as other psychiatric disorders (e.g., panic disorder and major depressive disorder [MDD]) or various other medical conditions, including cardiovascular, gastrointestinal, and respiratory diseases. ${ }^{3}$

Diabetes mellitus (DM), which has a global prevalence of $8.3 \%$, is a chronic and disabling disease and is a major cause of lost disability-adjusted life years. ${ }^{4,5} \mathrm{DM}$ and its complications impose a heavy burden not only on a personal level, but on a global level, i.e., on public health

Correspondence: Ching-Hua Lin, Kaohsiung Municipal Kai-Syuan Psychiatric Hospital, 130, Kai-Syuan 2nd Rd., Ling-Ya District, Kaohsiung 802, Taiwan.

E-mail: chua.lin@msa.hinet.net

Submitted Jul 12 2019, accepted Jan 06 2020, Epub Apr 172020. care systems. The number of DM patients is expected to increase faster in Asia than on other continents. ${ }^{5,6}$ Type 2 diabetes mellitus (T2DM) is now a major public health threat for ethnic Chinese populations in mainland China, Hong Kong, Taiwan, and Singapore, with adult prevalence rates having reached $20 \%$, $^{5,7}$

Co-occurring mental and physical illnesses, often categorized as chronic illness with complexity, is an emerging research area. Chronic illness with complexity is often defined as multiple chronic conditions occurring concurrently, regardless of causal pathways and associations. $^{8-10}$ However, mental illnesses that co-occur with physical illnesses are often considered discordant ${ }^{10-12}$ due to the special challenges of self-management and the varying treatment regimens for the co-occurring diseases. Conditions commonly observed in DM patients include clinical depression, anxiety disorder (AD), depressive affect, and diabetes-specific distress, all of which have

How to cite this article: Huang C-J, Hsieh H-M, Tu H-P, Jiang H-J, Wang P-W, Lin C-H. Generalized anxiety disorder in type 2 diabetes mellitus: prevalence and clinical characteristics. Braz J Psychiatry. 2020;42:621-629. http://dx.doi.org/10.1590/1516-4446-2019-0605 
been linked to negative effects on various bio-behavioral variables, including disease management, health care costs, days of missed work, and mortality. ${ }^{10,13-17}$ Research on the prevalence of GAD in T2DM patients is scant; most studies of the prevalence of co-occurring anxiety symptoms and $A D$ have focused on DM patients. ${ }^{18-21}$ Furthermore, these studies have focused on symptoms or self-reported measures rather than clinical diagnoses. ${ }^{18-21}$

Despite the rapid westernization of food and lifestyles in Asia, ethnic Chinese and Western societies differ widely in genetic factors, obesity, diet, culture, lifestyle, and medical resources. Although analyzing cultures is a critical component of epidemiology, research focusing on Asian populations, especially ethnic Chinese populations, is scant. Specifically, no comprehensive epidemiological studies on GAD in T2DM patients have been conducted in Taiwan. Thus, this study used Taiwan's National Health Insurance (NHI) database to estimate the prevalence of GAD in T2DM patients in order to obtain information needed for public health promotion efforts. This study first investigated the prevalence of GAD in patients treated for T2DM between 2000 and 2010 and then compared GAD-associated factors between T2DM patients and the general population (GP). Finally, factors associated with T2DM were analyzed in GAD patients.

\section{Methods}

\section{Data source}

The Taiwanese NHI program is a mandatory, single-payer system established in 1995; approximately $98 \%$ of Taiwan residents are enrolled in the $\mathrm{NHI}$ program, and almost all medical care providers in Taiwan, including those employed at medical and primary care centers, are contracted by the $\mathrm{NHI}$ Administration to provide outpatient and inpatient services. Through a fee-for-service payment system, all health care providers file monthly service claims to the $\mathrm{NHI}$ to receive reimbursements for their medical fees. These claims records include inpatient, ambulatory, and home care visits and associated information, such as patient demographic characteristics, clinical details, and health care utilization and expenditures.

\section{Sample}

This retrospective observational study analyzed a random sample of patients from the entire population of $\mathrm{NHI}$ enrollees from 2000 to 2010. In 2010, the NHI program provided medical claims data for 1 million randomly selected people (approximately $4.5 \%$ of Taiwan's population of 23 million) who were enrolled in the $\mathrm{NHI}$ and utilized health services in 2010. For these 1 million NHI enrollees, the registration and claims data constitute the Longitudinal Health Insurance Database 2010 (LHID 2010). The sample does not significantly differ from other enrollees in terms of age, gender, and average insured payroll-related amount. A random sample of $\mathrm{NHI}$ enrollees aged $\geqslant 20$ years each year during 2000-2010 were included.

\section{Definitions of T2DM and GAD}

Taiwanese NHI claims data include ICD-9-CM diagnostic codes. ${ }^{22}$ These data provided a useful structure for using ICD-9-CM diagnostic codes to identify T2DM patients and GAD. This study analyzed patients who had at least two service claims for ambulatory care or one service claim for inpatient care for a principal diagnosis of T2DM (ICD9CM codes 250.x0 and 250.x2). ${ }^{23,24} \mathrm{GAD}$ was defined as a record of at least one outpatient or inpatient service claim for a principal diagnosis of GAD (ICD9-CM code 300.02) between 2000 and 2010. ${ }^{17}$

\section{Prevalence of $G A D$}

The prevalence of GAD in the GP was calculated by dividing the number of GAD cases by the total GP. The prevalence of GAD in T2DM patients was calculated by dividing the number of GAD cases by the total number of T2DM patients.

\section{Measurements}

The patients' demographic characteristics, including age, gender, residence area, residence urbanization level, income, comorbidities, Charlson Comorbidity Index (CCl), and DM duration were obtained from each patient file retrieved from the $\mathrm{NHI}$ database. For the covariates listed in Table 1, we measured demographic characteristics based on the 2010 record. Patients were classified into seven age groups: 20-30, 31-40, 41-50, 51-60, 61-70, $71-80$, and $\geqslant 80$ years of age, while residence area was classified into five geographical regions of Taiwan: northern region, central region, southern region, eastern region, and offshore islets/other. Urbanization level was categorized as rural or urban. Average monthly income was classified into six categories: $\leqslant \mathrm{NT} \$ 17,280, \$ 17,281-\$ 22,880$, $\$ 22,881-\$ 28,800, \quad \$ 28,801-\$ 36,300, \quad \$ 36,301-\$ 45,800$, and $>\$ 45,800$. Comorbidities included myocardial infarction, congestive heart failure, peripheral vascular disease, hemiplegia or paraplegia, renal disease, cerebrovascular disease, and depressive disorder. The CCls were defined as 0 or $\geqslant 1$. The DM duration (years) was classified into four categories: $\leqslant 3,3-6$ (including the 6 th year), 6-9 (including the 9th year), and $>9$.

We also assessed patients who were prescribed oral antidiabetic therapy (ADT) or insulin injection therapy in at least three outpatient visits. Oral ADT was categorized into five groups: metformin (anatomical therapeutic chemical [ATC] code A10BA), sulfonylureas (ATC code A10BB), meglitinides (ATC code A10BX), thiazolidinediones (ATC code A10BG), or $\alpha$-glucosidase inhibitors (ATC code A10BF). Insulin injection therapy was classified as rapid-acting (ATC code $A 10 A B$ ), intermediateacting (ATC code A10AC), long-acting (ATC code A10AE), or a combination (ATC code A10AD).

\section{Statistical analysis}

The distribution of characteristics was compared among three groups of patients: T2DM with GAD, T2DM without 
Table 1 Demographic characteristics of type 2 diabetes mellitus patients with and without generalized anxiety disorder compared to the general population, 2010

\begin{tabular}{|c|c|c|c|c|c|c|}
\hline & $\begin{array}{l}\text { T2DM with } \\
\text { GAD }(n=645)\end{array}$ & $\begin{array}{l}\text { T2DM without } \\
\text { GAD }(n=6,722)\end{array}$ & $\begin{array}{c}\text { GP } \\
(n=715,756)\end{array}$ & P1 & P2 & P3 \\
\hline Age (years), mean (SD) & $63.5(11.4)$ & $62.5(13.2)$ & $44.5(16.0)$ & 0.0636 & $<0.0001$ & $<0.0001$ \\
\hline \multicolumn{7}{|l|}{ Age group } \\
\hline $20-30$ & $2(0.3)$ & $613(1.0)$ & $150,017(21.0)$ & & & \\
\hline $31-40$ & $12(1.9)$ & $2,404(3.9)$ & $168,180(23.5)$ & & & \\
\hline $41-50$ & $56(8.7)$ & $7,386(12.0)$ & $154,120(21.5)$ & & & \\
\hline $51-60$ & 189 (29.3) & $16,485(26.7)$ & $125,318(17.5)$ & & & \\
\hline $61-70$ & $187(29.0)$ & $16,087(26.1)$ & $59,114(8.3)$ & & & \\
\hline $71-80$ & $147(22.8)$ & $12,686(20.6)$ & $37,519(5.2)$ & & & \\
\hline$>80$ & $52(8.1)$ & $6,061(9.8)$ & $21,488(3.0)$ & 0.0008 & $<0.0001$ & $<0.0001$ \\
\hline \multicolumn{7}{|l|}{ Gender } \\
\hline Male & $224(34.7)$ & $31,043(50.3)$ & $345,736(48.3)$ & & & \\
\hline Female & $421(65.3)$ & $30,679(49.7)$ & $370,020(51.7)$ & $<0.0001$ & $<0.0001$ & $<0.0001$ \\
\hline \multicolumn{7}{|l|}{ Region } \\
\hline Northern & $300(46.5)$ & $28,177(45.7)$ & $343,067(47.9)$ & & & \\
\hline Central & $166(25.7)$ & $14,208(23.0)$ & $167,093(23.3)$ & & & \\
\hline Southern & $163(25.3)$ & $17,035(27.6)$ & $183,341(25.6)$ & & & \\
\hline Eastern & $15(2.3)$ & $1,681(2.7)$ & $16,174(2.3)$ & & & \\
\hline Offshore islets and other & $1(0.2)$ & $621(1.0)$ & $6,081(0.8)$ & 0.0776 & 0.2351 & $<0.0001$ \\
\hline \multicolumn{7}{|l|}{ Urbanization } \\
\hline Rural & $296(45.9)$ & $30,146(48.8)$ & $335,594(46.9)$ & & & \\
\hline Urban & $349(54.1)$ & $31,576(51.2)$ & $380,162(53.1)$ & 0.1359 & 0.6127 & $<0.0001$ \\
\hline \multicolumn{7}{|l|}{ Income (in NTD) } \\
\hline$\leqslant 17,280$ & $223(34.6)$ & $22,413(36.3)$ & $425,699(59.5)$ & & & \\
\hline $17,281-22,880$ & $303(47.0)$ & $25,573(41.4)$ & $172,793(24.1)$ & & & \\
\hline $22,881-28,800$ & $31(4.8)$ & $3,098(5.0)$ & $29,330(4.1)$ & & & \\
\hline $28,801-36,300$ & $23(3.6)$ & $3,712(6.0)$ & $32,903(4.6)$ & & & \\
\hline $36,301-45,800$ & $35(5.4)$ & $3,352(5.4)$ & $26,840(3.7)$ & & & \\
\hline$>45,800$ & $30(4.7)$ & $3,574(5.8)$ & $28,191(3.9)$ & 0.0221 & $<0.0001$ & $<0.0001$ \\
\hline \multicolumn{7}{|l|}{ Comorbidities } \\
\hline Myocardial infarction & $19(2.9)$ & $1,160(1.9)$ & $2,283(0.3)$ & 0.0479 & $<0.0001$ & $<0.0001$ \\
\hline Congestive heart failure & $98(15.2)$ & $6,380(10.3)$ & $14,821(2.1)$ & $<0.0001$ & $<0.0001$ & $<0.0001$ \\
\hline Peripheral vascular disease & $69(10.7)$ & $4,561(7.4)$ & $12,673(1.8)$ & 0.0014 & $<0.0001$ & $<0.0001$ \\
\hline Hemiplegia or paraplegia & $16(2.5)$ & $1,889(3.1)$ & $7,090(1.0)$ & 0.3946 & $<0.0001$ & $<0.0001$ \\
\hline Renal disease & $50(7.8)$ & $5,173(8.4)$ & $11,229(1.6)$ & 0.5661 & $<0.0001$ & $<0.0001$ \\
\hline Cerebrovascular disease & 167 (25.9) & $12,402(20.1)$ & $30,465(4.3)$ & $<0.0001$ & $<0.0001$ & $<0.0001$ \\
\hline Depression & $208(32.2)$ & $2,330(3.8)$ & $16,283(2.3)$ & $<0.0001$ & $<0.0001$ & $<0.0001$ \\
\hline $\mathrm{CCl}$, mean (SD) & $2.4(1.8)$ & $1.9(1.8)$ & $0.7(1.2)$ & $<0.0001$ & $<0.0001$ & $<0.0001$ \\
\hline \multicolumn{7}{|l|}{$\mathrm{CCl}$} \\
\hline 0 & $91(14.1)$ & $14,286(23.1)$ & $448,300(62.6)$ & & & \\
\hline$\geqslant 1$ & 554 (85.9) & $47,436(76.9)$ & $267,456(37.4)$ & $<0.0001$ & $<0.0001$ & $<0.0001$ \\
\hline \multicolumn{7}{|l|}{ Diabetes duration (years) } \\
\hline$\leqslant 3$ & $182(28.2)$ & $16,940(27.4)$ & & & & \\
\hline $3-6$ & $105(16.3)$ & $10,737(17.4)$ & & & & \\
\hline $6-9$ & 109 (16.9) & $11,604(18.8)$ & & & & \\
\hline$>9$ & $249(38.6)$ & $22,441(36.4)$ & & 0.4385 & & \\
\hline
\end{tabular}

Data presented as $\mathrm{n}(\%)$, unless otherwise specified.

Comorbidities and $\mathrm{CCl}$ for each comorbidity were defined as $\geqslant 3$ outpatient claims each.

$\mathrm{CCI}=$ Charlson Comorbidity Index; GAD = generalized anxiety disorder; GP = general population; NTD = New Taiwan dollar; P1 = T2DM with GAD vs. T2DM without GAD; P2 = T2DM with GAD vs. GP; P3 = T2DM without GAD vs. GP; SD = standard deviation; T2DM = type 2 diabetes mellitus.

GAD, and the GP. For categorical and continuous variables, $\chi 2$ and $t$-tests were used, respectively. Generalized linear mixed models, assuming a Poisson distribution, were used to compare the prevalence of GAD in T2DM patients and the GP. The risk factors considered in the estimates were age, gender, residence area, urbanization level, income, comorbidities, $\mathrm{CCl}$ and ADT. Prevalence ratios (PR) in the T2DM and GP groups were calculated and compared using a logbinomial model. A multiple logistic regression model was used to estimate the adjusted odds ratio (OR) and 95\% confidence interval $(95 \% \mathrm{Cl})$ to determine associations 
between GAD in T2DM patients and independent risk factors, including age, gender, residence area, urbanization level, income, comorbidities, $\mathrm{CCl}$, diabetes duration, and ADT. We also used the Cochran-Armitage test to determine trends for ordinal variables, such as income and diabetes duration. ${ }^{25,26}$ The Joinpoint Regression Program version 4.2.0.2 was used to estimate trends in GAD prevalence. The average annual percent change (AAPC) in GAD prevalence among T2DM patients or the GP was then estimated using joinpoint regression analysis, being a summary measure of the trend over a 1-year fixed interval. Statistical analyses were performed in SAS version 9.4. Statistical tests were double-sided, with $p$ values $<0.05$ considered statistically significant.

\section{Ethics statement}

This study was conducted according to Declaration of Helsinki guidelines and was approved by the institutional review board of Kaohsiung Medical University Hospital.

\section{Results}

In 2010 , a sample of 715,756 patients aged $\geqslant 20$ years were analyzed from the study database. In Table 1, the demographic characteristics for 2010, including age, gender, residence area, urbanization level, income, comorbidities, $\mathrm{CCl}$ score, and diabetes duration are compared among the T2DM with GAD group ( $n=645)$, T2DM without GAD group ( $n=61,722)$, and the GP group $(n=715,756)$. The T2DM with GAD group and the T2DM without GAD group significantly differed in all demographic characteristics except for mean age, residence area, urbanization level, comorbidities (hemiplegia or paraplegia, and renal disease), and diabetes duration. Except for region and urbanization, all demographic characteristics significantly differed between the GP group and the T2DM with GAD group. All demographic characteristics significantly differed between the GP group and the T2DM without GAD group.

Figure 1 compares temporal trends in GAD prevalence from 2000 to 2010. During this period, the prevalence of GAD increased from 0.81 to $1.03 \%$ in the T2DM group and from 0.25 to $0.63 \%$ in the GP group. GAD was significantly $(p<0.0001)$ more prevalent in the T2DM group than the GP group. The AAPC significantly differed from 0 at alpha $=0$; the AAPC for GAD in T2DM was $3.2 \%(95 \% \mathrm{Cl} 1.7-4.8, \mathrm{p}<0.05)$, and the AAPC for GAD in GP was $10.3 \%(95 \% \mathrm{Cl} 8.7-12.0, p<0.05)$. The AAPC for GAD was significantly higher in the GP than in the T2DM group (comparison, $-7.1,95 \% \mathrm{Cl}-9.3$ to -5.0 , $p<0.05)$. In 2010, however, the PR for GAD in the T2DM group compared to the GP group was $1.63(95 \% \mathrm{Cl}$ 1.50-1.77).

Table 2 compares the prevalence of GAD between the T2DM and GP groups in 2010. The 1-year prevalence rate for GAD was significantly higher in the T2DM group than the GP group (1.03 vs. $0.63 \%$; PR: $1.63 ; 95 \% \mathrm{Cl}$ 1.50-1.77; $p<0.0001$ ). The 1-year prevalence of GAD was higher in T2DM patients who had received ADT than the GP (0.90 vs. $0.63 \%$, respectively) and was higher in T2DM patients who had not received ADT than the GP ( 1.60 vs. $0.63 \%$, respectively). There was a higher prevalence of $A D$ in the T2DM group than the GP among individuals with the following demographic characteristics: age 51-60 years; men and women, all residence area other than eastern and offshore islets/other, both urban and rural areas; income $\leqslant$ NT\$ 17,280, \$17,281$\$ 22,880$, or $\$ 36,301-\$ 45,800$, or with CCls and a CCl score of 0 . However, individuals with comorbid renal disease and cerebrovascular disease in the T2DM group had a lower prevalence of GAD.

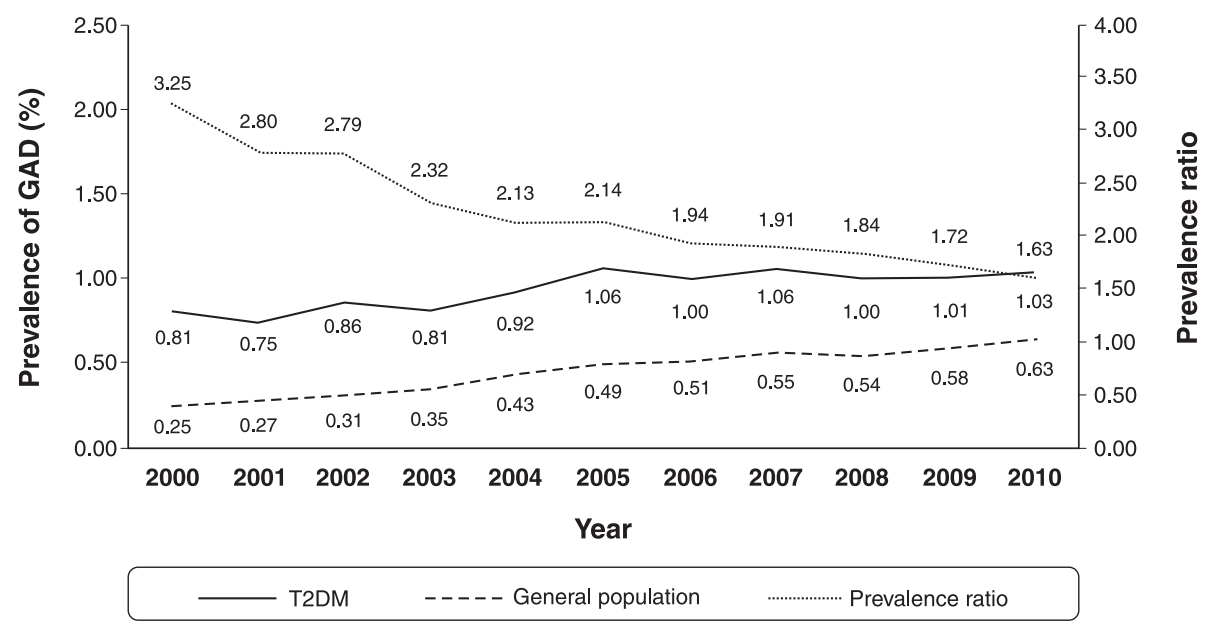

Figure 1 Prevalence of GAD in T2DM patients, in the GP, and GAD prevalence ratios. Temporal trend in GAD prevalence from 2000 to 2010. The prevalence of GAD was calculated by dividing the number of GAD cases by the total number of T2DM patients or GP. The rates increased from 0.81 to $1.03 \%$ in T2DM and from 0.25 to $0.63 \%$ in the GP. The prevalence of GAD in T2DM patients was significantly higher overall than in the GP from 2000 to $2010(\mathrm{p}<0.0001)$. Moreover, each year from 2000 to 2010 the prevalence ratios of GAD were significantly higher in T2DM patients than the GP $(p<0.0001)$. The T2DM-to-GP GAD prevalence ratio decreased from 3.25 in 2000 to 1.63 in 2010. GAD = generalized anxiety disorder; GP = general population; T2DM = type 2 diabetes mellitus. 
Table 2 Prevalence of generalized anxiety disorder and the prevalence ratio of type 2 diabetes mellitus, 2010

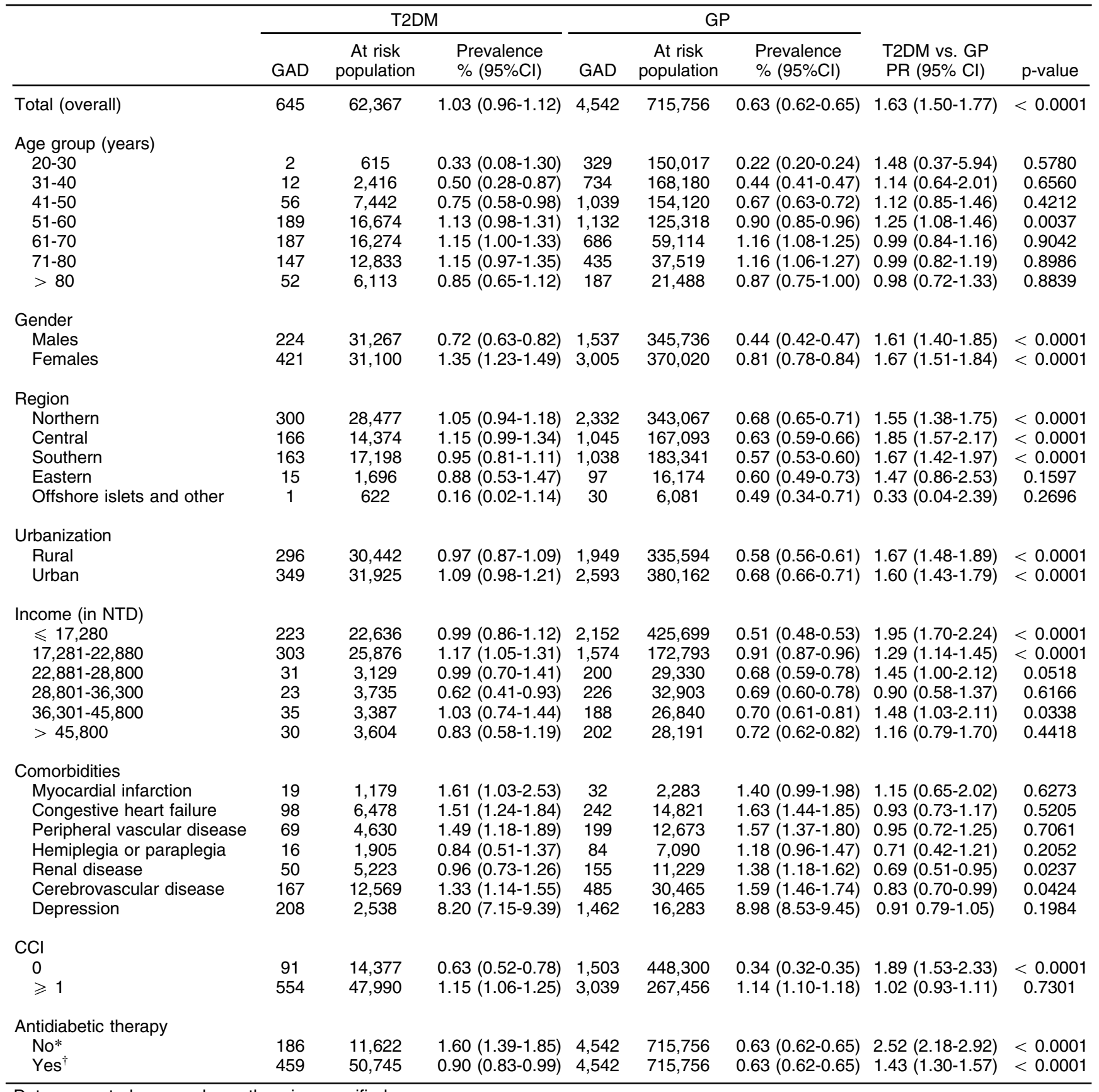

Data presented as $\mathrm{n}$, unless otherwise specified.

Comorbidities, $\mathrm{CCl}$ for each comorbidity, and antidiabetic therapy were defined as $\geqslant 3$ outpatient claims each.

PR with $95 \% \mathrm{Cl}$ was estimated with generalized linear mixed models, assuming a Poisson distribution.

PR with $95 \% \mathrm{Cl}$ was estimated with a log-binomial model.

$95 \% \mathrm{Cl}=95 \%$ confidence interval; $\mathrm{CCl}=$ Charlson Comorbidity Index; GAD = generalized anxiety disorder; GP = general population;

NTD = New Taiwan dollar; PR = prevalence ratio; T2DM = type 2 diabetes mellitus.

* T2DM patients without antidiabetic therapy compared to the GP.

T2DM patients with antidiabetic therapy compared to the GP.

Table 3 shows the results of the multiple logistic regression analysis for factors associated with the prevalence of GAD in T2DM patients. The prevalence of GAD in T2DM patients was associated with female gender, $\mathrm{CCl} \geqslant 1$, DM duration $>9$ years, congestive heart failure, peripheral vascular disease, and depressive disorder. Results from the Cochran-Armitage trend test indicated there were no statistical trends for income and diabetes duration associated with the prevalence of GAD. The prevalence of GAD was low in T2DM patients who used metformin, sulfonylureas, and rapid-acting insulin injection therapy. Notably, the highest prevalence of GAD was associated with comorbid depressive disorder (OR = 10.71; 95\% Cl 8.99-12.76; $p<0.0001$ ). 
Table 3 Adjusted odds ratio for the prevalence of generalized anxiety disorder in type 2 diabetes mellitus patients

\begin{tabular}{|c|c|c|}
\hline & $\begin{array}{l}\text { Adjusted OR } \\
(95 \% \mathrm{Cl})\end{array}$ & $\mathrm{p}$-value \\
\hline \multicolumn{3}{|l|}{ Age group (years) } \\
\hline $20-30$ & 1.00 & \\
\hline $31-40$ & $1.42(0.31-6.40)$ & 0.6519 \\
\hline $41-50$ & $2.24(0.54-9.31)$ & 0.2688 \\
\hline $51-60$ & $3.20(0.78-13.12)$ & 0.1064 \\
\hline $61-70$ & $3.03(0.74-12.46)$ & 0.1244 \\
\hline $71-80$ & $2.71(0.66-11.20)$ & 0.1674 \\
\hline$>80$ & $1.91(0.46-8.02)$ & 0.3763 \\
\hline \multicolumn{3}{|l|}{ Gender } \\
\hline Male & 1.00 & \\
\hline Female & $1.59(1.33-1.89)$ & $<0.000$ \\
\hline \multicolumn{3}{|l|}{ Region } \\
\hline Northern & 1.00 & \\
\hline Central & $1.16(0.92-1.45)$ & 0.2010 \\
\hline Southern & $0.92(0.75-1.13)$ & 0.4149 \\
\hline Eastern & $0.87(0.51-1.51)$ & 0.6313 \\
\hline Offshore islets and other & $0.16(0.02-1.14)$ & 0.0666 \\
\hline \multicolumn{3}{|l|}{ Urbanization } \\
\hline Rural & 1.00 & \\
\hline Urban & $1.20(0.99-1.45)$ & 0.0567 \\
\hline \multicolumn{3}{|l|}{ Income (in NTD) } \\
\hline$\leqslant 17,280$ & $0.95(0.63-1.42)$ & 0.7888 \\
\hline $17,281-22,880$ & $1.12(0.75-1.66)$ & 0.5831 \\
\hline $22,881-28,800$ & $1.01(0.60-1.69)$ & 0.9676 \\
\hline $28,801-36,300$ & $0.66(0.38-1.15)$ & 0.1429 \\
\hline $36,301-45,800$ & $1.09(0.66-1.79)$ & 0.7388 \\
\hline$>45,800$ & 1.00 & \\
\hline \multicolumn{3}{|l|}{ Comorbidities } \\
\hline Myocardial infarction & $1.57(0.97-2.53)$ & 0.0640 \\
\hline Congestive heart failure & $1.36(1.07-1.71)$ & 0.0103 \\
\hline Peripheral vascular disease & $1.30(1.00-1.69)$ & 0.0480 \\
\hline Hemiplegia or paraplegia & $0.63(0.38-1.05)$ & 0.0789 \\
\hline Renal disease & $0.79(0.58-1.08)$ & 0.1399 \\
\hline Cerebrovascular disease & $1.18(0.97-1.44)$ & 0.0926 \\
\hline Depression & $10.71(8.99-12.76)$ & $<0.0001$ \\
\hline \multicolumn{3}{|l|}{$\mathrm{CCl}$} \\
\hline 0 & 1.00 & \\
\hline$\geqslant 1$ & $1.32(1.04-1.68)$ & 0.0218 \\
\hline \multicolumn{3}{|l|}{ Diabetes duration (years), n (\%) } \\
\hline$\leqslant 3$ & 1.00 & \\
\hline $3-6$ & $1.11(0.86-1.44)$ & 0.4242 \\
\hline $6-9$ & $1.08(0.83-1.40)$ & 0.5792 \\
\hline$>9$ & $1.29(1.00-1.66)$ & 0.0463 \\
\hline \multicolumn{3}{|l|}{ Oral antidiabetic therapy } \\
\hline Metformin (A10BA) & $0.73(0.59-0.91)$ & 0.0042 \\
\hline Sulfonylureas (A10BB) & $0.67(0.54-0.84)$ & 0.0004 \\
\hline Meglitinides (A10BX) & $1.08(0.85-1.38)$ & 0.5226 \\
\hline Thiazolidinediones (A10BG) & $1.09(0.87-1.36)$ & 0.4524 \\
\hline$\alpha$-glucosidase inhibitor (A10BF) & $0.96(0.76-1.19)$ & 0.6905 \\
\hline \multicolumn{3}{|l|}{ Insulin injection therapy } \\
\hline Rapid-acting (A10AB), & $0.65(0.44-0.95)$ & 0.0276 \\
\hline Intermediate-acting (A10AC) & $1.22(0.77-1.92)$ & 0.3998 \\
\hline Long-acting (A10AE) & $1.04(0.64-1.68)$ & 0.8710 \\
\hline Combination (A10AD) & $1.27(0.81-1.99)$ & 0.2903 \\
\hline
\end{tabular}

Comorbidities, $\mathrm{CCl}$ for each comorbidity, oral antidiabetic therapy, and insulin injection therapy for each ATC code were defined as $\geqslant 3$ outpatient claims each.

$95 \% \mathrm{Cl}=95 \%$ confidence interval; ATC = Anatomical Therapeutic Chemical; $\mathrm{CCl}=$ Charlson Comorbidity Index; NTD = New Taiwan dollar; OR $=$ odds ratio.

\section{Discussion}

This study is the first to use the population-based $\mathrm{NHI}$ dataset to estimate the prevalence of GAD in T2DM patients and the GP of Taiwan. Because the NHI program covers $98 \%$ of Taiwan's population, the prevalence data obtained from this study approximate the actual distribution of GAD in Taiwanese T2DM patients. To the best of our knowledge, little or no real-world data are available for GAD diagnoses in T2DM patients. Most studies of T2DM patients have focused on the prevalence of anxiety symptoms or $A D$, rather than on GAD. ${ }^{11,17-21,27-32}$ Another unique feature of this study is that it analyzed a specific Asian population (i.e., ethnic Han Chinese).

Table 1 shows that, compared to GAD patients in the GP, T2DM GAD patients were more likely to have high $\mathrm{CCl}$ scores, be older, female, and have multiple comorbidities. Figure 1 shows that from 2000 to 2010, the prevalence of GAD was significantly higher in the T2DM group than the GP group, which is consistent with the results of other studies on anxiety symptoms or $A D$ in Western populations. ${ }^{29,32}$ Between 2000 and 2010, the prevalence of GAD increased in both the T2DM group and the GP group. During this period, the AAPC in GAD was $3.2 \%$ in the T2DM group and $10.3 \%$ in the GP group. Table 2 shows that in 2010 the AAPC in GAD was larger in the GP group than in the T2DM group. However, the $P R$ of $G A D$ in the T2DM group vs. the GP was 1.63 . Between 2000 and 2010, the number of GAD cases increased in both the T2DM group (from 209 to 645) and the GP group (from 1,875 to 4,542). Furthermore, the number of T2DM cases increased from 25,827 in 2000 to 62,367 in 2010, although the number decreased from 752,296 in 2000 to 715,756 in 2010 in the GP. This explains why the AAPC in GAD was larger in the GP group than the T2DM group. Another possible explanation for this difference is that the T2DM group received more comprehensive medical support than the GP. Better medical support could reduce anxiety, ${ }^{33}$ which is consistent with the annual medical support burden growth among T2DM patients in Taiwan. ${ }^{34}$ Table 2 shows that the 1-year prevalence of GAD in 2010 was higher in the T2DM group (1.03\%) than the GP group $(0.63 \%)$, which is consistent with the results of $A D$ studies performed in Western countries. ${ }^{29,32}$ A systematic review reported that, in the GP of western countries, the median 12-month GAD prevalence was $3.9 \%$ (range, 2.1-6.6\%) and that the median lifetime prevalence of GAD was $12 \%$ (range, $8-13.7 \%){ }^{2}$ A Chinese study reported that the overall 12-month and lifetime prevalence of GAD were 0.8 and $1.2 \%$, respectively. ${ }^{35}$ In the GP group analyzed in the current study, the 1-year prevalence of GAD in 2010 $(0.63 \%)$ was much lower than that reported in western countries, but was comparable to that reported in China. $^{2,35}$ Our literature review only included studies on $A D$, not studies on GAD in T2DM. The 1-year prevalence of any diagnosed $A D$ in T2DM patients is $14 \%{ }^{32}$

Table 3 shows that the prevalence of GAD was not associated with age in the T2DM patients analyzed in this study. Previous studies have reported an association between younger or middle-aged T2DM patients and a 
high prevalence of $\mathrm{AD} .^{18,19,29,30,36}$ However, the data used in most of the studies performed so far have been limited to symptom ratings or data collected by telephone surveys rather than data on clinical diagnoses of $A D$. The current study also found a higher GAD risk in female T2DM patients than their male counterparts, which is consistent with reports that the co-occurrence of $A D$ and diabetes is higher in women than in men. ${ }^{8,18,20,28-30,32}$ Possible causes for this higher prevalence are hormonal differences and the effects of childbirth. Other possible causes are gender differences in the response to psychosocial stressors and major life events (such as the loss of a spouse), gender differences in social and family roles (such as being the primary caregiver in a household), and gender differences in the occurrence of chronic diseases and conditions. ${ }^{10}$ Further systematic studies are needed to explore biological, behavioral, and psychological mechanisms underlying the higher prevalence of GAD in women.

Notably, the prevalence of GAD in this sample of T2DM patients did not significantly differ by geographic region or by urbanization level. To date, no consistent findings on the relative prevalence of $A D$ rural and urban areas have been reported. ${ }^{17,21}$ Furthermore, there was no significant difference in income and GAD prevalence among T2DM patients in this study. Nevertheless, low income is a reported risk factor for increased depressive and anxiety symptoms in diabetes patients. ${ }^{17,31,37}$

Multivariate analysis of T2DM patients revealed that GAD risk was significantly associated with most of the comorbidities considered in this study, including congestive heart failure, peripheral vascular disease, depressive disorder, and multiple comorbidities $(\mathrm{CCl} \geqslant 1)$. Depressive disorder was a major risk factor for GAD in T2DM patients, which is consistent with the literature. ${ }^{28}$ Other comorbidities were not associated with GAD in T2DM patients, although depressive disorder was probably a major confounding factor in associations with GAD. There was a high risk of GAD among patients with a long duration ( $>9$ years) of T2DM, which is consistent with reports that a long duration of $D M$ is associated with a high risk of $A D$ and with worsening anxiety symptoms. ${ }^{27,28}$

Of the T2DM patients in this study, those with the highest GAD risk had comorbid depressive disorder, which is consistent with the literature. ${ }^{28}$ Another study reported that MDD patients and GAD patients often exhibit symptoms of both disorders simultaneously. ${ }^{29}$ Thus, clinicians should compare diagnostic criteria for both conditions and be aware that co-occurring depressive disorder and GAD require more complex and intensive treatment than either of these disorders occurring alone.

Diabetes management usually starts with lifestyle interventions, followed by treatment with a single medication (metformin, sulfonylureas, meglitinide, thiazolidinedione, and $\alpha$-glucosidase inhibitors), treatment with multiple medications or a single basal insulin injection and, finally, treatment with multiple insulin injections. Use of metformin and sulfonylureas were associated with significantly lower GAD rates among the T2DM patients in this study. The anxiolytic effect of glucose stabilization remains controversial. ${ }^{38,39} \mathrm{~A}$ study by Sarkaki et al. ${ }^{38}$ indicated that metformin may improve anxiety-like behaviors through AMPK-dependent regulation of autophagy following transient forebrain ischemia. However, further studies are needed to clarify whether the aforementioned oral ADTs are associated with GAD in the context of T2DM. Insulin can be categorized as rapid-acting, intermediate-acting, or long-acting. Injections of one of these types, or a combination of these types, are the most effective therapy after failed oral ADT therapy. Our analysis showed that GAD was only associated with rapid-acting insulin, which conferred a low risk of GAD in T2DM patients. Although an association between anxiety symptoms and insulin therapy has been established, ${ }^{40}$ animal models of streptozotocininduced diabetes have revealed that improved dysregulation of neurotransmitters after insulin injection has an anxiolytic effect. ${ }^{41} \mathrm{~A}$ recent study also reported that insulin has a protective effect against $A D$ after multiple risk adjustments. ${ }^{28}$ Further studies are needed to clarify the associations between each insulin type and GAD in T2DM patients.

This study estimated the prevalence of GAD in a large, randomly selected, population-based sample of T2DM patients and the GP. Insurance data are useful for studying GAD in T2DM patients because of the large number of patients available for data sampling. Using a health insurance database avoids the need to spend time and money performing psychiatric assessments and collecting longitudinal data on GAD prevalence and associated risk factors. ${ }^{10,42}$ However, the limitations of a database study include the potential for inconsistencies in the diagnostic criteria for GAD and T2DM, reduced reliability and validity of secondary data, ${ }^{10,24}$ dual diagnoses, and over- and underdiagnoses. ${ }^{10,43}$ In patients with multiple comorbidities, the coding order can also introduce complexities. However, since this study limited the maximum number of diagnostic codes to five for each admission or outpatient visit, the order of diagnostic codes probably did not affect the results of this study. That is, diagnostic codes were analyzed for all patients except those with $>5$ comorbidities. Another limitation was that data for certain essential variables were not accessed, including education level, occupation, marital status, lifestyle factors, physical activity, blood glucose control, glycemic level, and body weight. Additionally, this study did not calculate the accumulated dosage of medications, insulin, or antidepressants. Furthermore, the sequence of occurrence of T2DM and GAD was not recorded. Finally, causalities could not be fully clarified. Causal relationships between the high comorbidity of GAD in T2DM patients could not be established without knowing whether T2DM is a risk factor for GAD or the reverse. Thus, further research is needed to explore causation between the prevalence of T2DM and GAD. Studies on the prevalence of T2DM and GAD must be carefully designed and select the appropriate measurement instruments. Regular follow-up studies of $\mathrm{NHI}$ data are needed to compare the accumulating epidemiologic data for patients in Taiwan.

In conclusion, this study had several key findings. The prevalence of GAD was significantly higher in T2DM patients than in the GP between 2000 and 2010. In 2010, 
the 1-year prevalence of GAD in the GP was much lower than that in western countries but was comparable to that of China. ${ }^{2,35}$ The prevalence of GAD increased from 0.81 to $1.03 \%$ in T2DM patients during the study period. Compared to patients with GAD in the GP, T2DM patients with GAD tended to be female, older, and have more comorbidities and higher CCls. From 2000 to 2010, the prevalence of GAD increased in both the T2DM patients and the GP. Specifically, the high prevalence of GAD in T2DM patients was associated with female gender, $\mathrm{CCl}$ $\geqslant 1$, DM duration $>9$ years, comorbid congestive heart failure, peripheral vascular disease, and depressive disorder. T2DM patients who received metformin, sulfonylureas, and rapid-acting insulin injection therapy had a low GAD prevalence. The highest risk factor for GAD in T2DM patients was comorbid depressive disorder. These results suggest that physicians should carefully screen T2DM patients for GAD, particularly those who exhibit the above risk factors. Public health officials must also develop effective prevention and treatment strategies for T2DM patients who have a high risk for GAD, particularly those with comorbid depressive disorder.

\section{Acknowledgements}

This study was supported by a grant from Kaohsiung Medical University Hospital (KMUH105-5T08). This study is based in part on data from the NHI Research Database provided by the Bureau of $\mathrm{NHI}$, Department of Health, and managed by National Health Research Institutes (NHIRD100-100 and NHIRD-102-135). The interpretation and conclusions contained herein do not represent those of the aforementioned agencies.

\section{Disclosure}

The authors report no conflicts of interest.

\section{References}

1 de Dios MA, Hagerty CE, Herman DS, Hayaki J, Anderson BJ, Budney AJ, et al. General anxiety disorder symptoms, tension reduction, and marijuana use among young adult females. J Womens Health (Larchmt). 2010;19:1635-42.

2 Haller H, Cramer H, Lauche R, Gass F, Dobos GJ. The prevalence and burden of subthreshold generalized anxiety disorder: a systematic review. BMC Psychiatry. 2014;14:128.

3 Doria S, de Vuono A, Sanlorenzo R, Irtelli F, Mencacci C. Anti-anxiety efficacy of Sudarshan Kriya Yoga in general anxiety disorder: a multicomponent, yoga based, breath intervention program for patients suffering from generalized anxiety disorder with or without comorbidities. J Affect Disord. 2015;184:310-7.

4 Murray CJ, Vos T, Lozano R, Naghavi M, Flaxman AD, Michaud C, et al. Disability-adjusted life years (DALYs) for 291 diseases and injuries in 21 regions, 1990-2010: a systematic analysis for the global burden of disease study 2010. Lancet. 2012;380:2197-223.

5 Lin WH, Hsu CH, Chen HF, Liu CC, Li CY. Mortality of patients with type 2 diabetes in Taiwan: a 10-year nationwide follow-up study. Diabetes Res Clin Pract. 2015;107:178-86.

6 Amos AF, McCarty DJ, Zimmet P. The rising global burden of diabetes and its complications: estimates and projections to the year 2010. Diabet Med. 1997;14 Suppl 5: S1-85.

7 Gu D, Reynolds K, Duan X, Xin X, Chen J, Wu X, et al. Prevalence of diabetes and impaired fasting glucose in the Chinese adult population: international collaborative study of cardiovascular disease in Asia (InterASIA). Diabetologia. 2003;46:1190-8.
8 Banerjea R, Pogach LM, Smelson D, Sambamoorthi U. Mental illness and substance use disorders among women veterans with diabetes. Womens Health Issues. 2009;19:446-56.

9 Werner RM, Greenfield S, Fung C, Turner BJ. Measuring quality of care in patients with multiple clinical conditions: summary of a conference conducted by the society of general internal medicine. J Gen Intern Med. 2007;22:1206-11.

10 Huang CJ, Lin $\mathrm{CH}$, Lee MH, Chang KP, Chiu HC. Prevalence and incidence of diagnosed depression disorders in patients with diabetes: a national population-based cohort study. Gen Hosp Psychiatry. 2012;34:242-8.

11 Banerjea R, Sambamoorthi U, Smelson D, Pogach LM. Chronic illness with complexities: mental illness and substance use among veteran clinic users with diabetes. Am J Drug Alcohol Abuse. 2007;33:807-21.

12 Piette JD, Kerr EA. The impact of comorbid chronic conditions on diabetes care. Diabetes Care. 2006;29:725-31.

13 Ciechanowski PS, Katon WJ, Russo JE. Depression and diabetes: impact of depressive symptoms on adherence, function, and costs. Arch Intern Med. 2000;160:3278-85.

14 Egede LE, Zheng D, Simpson K. Comorbid depression is associated with increased health care use and expenditures in individuals with diabetes. Diabetes Care. 2002;25:464-70.

15 Egede LE. Effects of depression on work loss and disability bed days in individuals with diabetes. Diabetes Care. 2004;27:1751-3.

16 Katon WJ, Rutter C, Simon G, Lin EH, Ludman E, Ciechanowski P, et al. The association of comorbid depression with mortality in patients with type 2 diabetes. Diabetes Care. 2005;28:2668-72.

17 Huang CJ, Chiu HC, Lee MH, Wang SY. Prevalence and incidence of anxiety disorders in diabetic patients: a national population-based cohort study. Gen Hosp Psychiatry. 2011;33:8-15.

18 Collins MM, Corcoran P, Perry IJ. Anxiety and depression symptoms in patients with diabetes. Diabet Med. 2009;26:153-61.

19 Peyrot M, Rubin RR. Levels and risks of depression and anxiety symptomatology among diabetic adults. Diabetes Care. 1997;20: 585-90.

20 Lloyd $\mathrm{CE}$, Dyer $\mathrm{PH}$, Barnett $\mathrm{AH}$. Prevalence of symptoms of depression and anxiety in a diabetes clinic population. Diabet Med. 2000;17:198-202.

21 Camara A, Balde NM, Enoru S, Bangoura JS, Sobngwi E, Bonnet F. Prevalence of anxiety and depression among diabetic African patients in Guinea: association with $\mathrm{HbA1c}$ levels. Diabetes Metab. 2015;41:62-8.

22 Chinese Hospital Association. ICD-9-CM English-Chinese Dictionary. Taipei: Chinese Hospital Association Press; 2000.

23 Solberg LI, Engebretson KI, Sperl-Hillen JM, Hroscikoski MC, O'Connor PJ. Are claims data accurate enough to identify patients for performance measures or quality improvement? The case of diabetes, heart disease, and depression. Am J Med Qual. 2006;21:238-45.

24 Lin CC, Lai MS, Syu CY, Chang SC, Tseng FY. Accuracy of diabetes diagnosis in health insurance claims data in Taiwan. J Formos Med Assoc. 2005;104:157-63.

25 Armitage P. Tests for linear trends in proportions and frequencies. Biometrics. International Biometric Society. Biometrics. 1955;11: 375-86.

26 Cochran WG. Some methods for strengthening the common chisquared tests. Biometrics. 1954;10:417-51.

27 Trento M, Charrier L, Salassa M, Merlo S, Passera P, Cavallo F, et al. Depression, anxiety and cognitive function in patients with type 2 diabetes: an 8-year prospective observational study. Acta Diabetol. 2015;52:1157-66.

28 Mikaliukstiene A, Zagminas K, Juozulynas A, Narkauskaite L, Salyga $\mathrm{J}$, Jankauskiene $\mathrm{K}$, et al. Prevalence and determinants of anxiety and depression symptoms in patients with type 2 diabetes in Lithuania. Med Sci Monit. 2014;20:182-90.

29 Fisher L, Skaff MM, Mullan JT, Arean P, Glasgow R, Masharani U. A longitudinal study of affective and anxiety disorders, depressive affect and diabetes distress in adults with type 2 diabetes. Diabet Med. 2008;25:1096-101.

30 Hermanns N, Kulzer B, Krichbaum M, Kubiak T, Haak T. Affective and anxiety disorders in a German sample of diabetic patients: prevalence, comorbidity and risk factors. Diabet Med. 2005;22:293-300.

31 Thomas J, Jones G, Scarinci I, Brantley P. A descriptive and comparative study of the prevalence of depressive and anxiety disorders 
in low-income adults with type 2 diabetes and other chronic illnesses. Diabetes Care. 2003;26:2311-7.

32 Grigsby AB, Anderson RJ, Freedland KE, Clouse RE, Lustman PJ. Prevalence of anxiety in adults with diabetes: a systematic review. J Psychosom Res. 2002;53:1053-60.

33 A 5-year randomized controlled study of learning, problem solving ability, and quality of life modifications in people with type 2 diabetes managed by group care. Diabetes Care. 2004;27:670-5.

34 Wang CY, Yu NC, Sheu WH, Tsai ST, Tai TY. Team care of type 2 diabetes mellitus in Taiwan. Diabetes Res Clin Pract. 2014;106 Suppl 2: S309-13.

35 Ma X, Xiang YT, Cai ZJ, Lu JY, Li SR, Xiang YQ, et al. Generalized anxiety disorder in China: prevalence, sociodemographic correlates, comorbidity, and suicide attempts. Perspect Psychiatr Care. 2009;45: 119-27.

36 Li C, Barker L, Ford ES, Zhang X, Strine TW, Mokdad AH. Diabetes and anxiety in US adults: findings from the 2006 behavioral risk factor surveillance system. Diabet Med. 2008;25:878-81.

37 Kahn RS, Wise PH, Kennedy BP, Kawachi I. State income inequality, household income, and maternal mental and physical health: cross sectional national survey. BMJ. 2000;321:1311-5.
38 Sarkaki A, Farbood Y, Badavi M, Khalaj L, Khodagholi F, Ashabi G. Metformin improves anxiety-like behaviors through AMPK-dependent regulation of autophagy following transient forebrain ischemia. Metab Brain Dis. 2015;30:1139-50.

39 Bodmer M, Meier C, Krahenbuhl S, Jick SS, Meier CR. Metformin, sulfonylureas, or other antidiabetes drugs and the risk of lactic acidosis or hypoglycemia: a nested case-control analysis. Diabetes Care. 2008;31:2086-91.

40 Zambanini A, Newson RB, Maisey M, Feher MD. Injection related anxiety in insulin-treated diabetes. Diabetes Res Clin Pract. 1999;46: 239-46.

41 Gupta D, Radhakrishnan M, Kurhe Y. Insulin reverses anxiety-like behavior evoked by streptozotocin-induced diabetes in mice. Metab Brain Dis. 2014;29:737-46.

42 Chien IC, Chou YJ, Lin CH, Bih SH, Chou P, Chang HJ. Prevalence and incidence of schizophrenia among national health insurance enrollees in Taiwan, 1996-2001. Psychiatry Clin Neurosci. 2004;58: 611-8.

43 Liptzin B, Regier DA, Goldberg ID. Utilization of health and mental health services in a large insured population. Am $J$ Psychiatry. 1980;137:553-8. 